\title{
Lugares favoritos no envelhecimento: Explorando estudos e conceitos
}

\author{
Favorite places in aging: Exploring studies and concepts
}

Mariana Lopez ${ }^{[\mathrm{[a]}}$, Maíra Longhinotti Felippe ${ }^{[b]}$, Ariane Kuhnen ${ }^{[c]}$

${ }^{[a]}$ Psicóloga, pós-graduada em Neuropsicologia Clínica, mestranda do Programa de Pós-Graduação em Psicologia da Universidade Federal de Santa Catarina (UFSC), Florianópolis, SC - Brasil, e-mail: psicolopez@gmail.com

${ }^{[b]}$ Arquiteta e Urbanista, mestranda do Programa de Pós-Graduação em Psicologia da Universidade Federal de Santa Catarina (UFSC), Florianópolis, SC - Brasil, e-mail: mairafelippe@gmail.com

[c] Psicóloga, mestre em Sociologia Política e doutora em Ciências Humanas, professora do Departamento de Psicologia e do Programa de Pós-Graduação em Psicologia da Universidade Federal de Santa Catarina (UFSC), Florianópolis, SC - Brasil, e-mail: ariane@cfh.ufsc.br

Recebido: 05/05/2010 Received: 05/05/2010

Aprovado: 17/03/2011 Approved: 03/17/2011

\section{Resumo}

Este trabalho de revisão bibliográfica busca apresentar e discutir a relação pessoa-ambiente no processo do envelhecimento, sobretudo em relação ao lugar favorito. Para isso, foi realizada uma busca na base de dados BVS-PSI e no Journal of Environmental Psychology com as palavras-chave: lugares favoritos e envelhecimento; lugares favoritos e idosos; favorite places e aging - em julho de 2009. Dois objetivos centrais foram contemplados: apresentar os documentos que aparecem com as palavras de busca utilizadas; discutir aqueles documentos que mencionam no corpo do texto a relação lugar/lugares favoritos no envelhecimento, explorando o conteúdo dos mesmos. Foi encontrado apenas um documento na BVS-PSI e 13 no Journal of Environmental Psychology, sendo selecionados para discussão sete documentos. Identidade de lugar, lugar favorito e capacidade restauradora dos lugares foram conceitos presentes em todos os trabalhos. Observou-se que a possibilidade de interações sociais e o ambiente da casa geralmente descrevem os lugares favoritos do idoso, estimulando a experiência de bem-estar e restauração decorrente da satisfação e do prazer vivenciado. Como forma de avaliar a contribuição teórico-metodológica destes trabalhos à Psicologia Ambiental, também foram observadas questões relativas à constituição disciplinar das equipes de pesquisadores, bem como as perspectivas teóricas, os paradigmas de investigação, os delineamentos metodológicos e os contextos de pesquisa - se naturais ou artificiais - adotados nestes estudos.

Palavras-chave: Lugar. Lugar favorito. Envelhecimento. 
the experience of well being and restoration arising from the satisfaction and pleasure experienced. As a way of assessing the theoretical and methodological contribution of these works to Environmental Psychology, issues relating to disciplinary training of researchers, as well as theoretical perspectives, research paradigms, methodological outline and research contexts whether natural or artificial - adopted in these studies were also analyzed.

Keywords: Place. Favorite place. Aging.

\section{Introducãa}

Atualmente é crescente o número de idosos no Brasil. Entre os anos de 1991 e 2000, o número de pessoas com 60 anos ou mais aumentou $35 \%$, ou seja, 2,5 vezes mais que as demais faixas etárias do país, que cresceram apenas 14\% (Aversi-Ferreira, Rodrigues \& Paiva, 2008). Essa fase do desenvolvimento implica mudanças nos processos psicológicos e fisiológicos do organismo, acarretando adaptações no contexto social e nas ressignificações ambientais da relação de apego e desapego com lugares (Aversi-Ferreira, Rodrigues \& Paiva, 2008; Günther, Nepomuceno, Spehar \& Günther, 2003).

Estudos que englobam conhecimentos da Psicologia Ambiental relacionando-os à Psicologia do Desenvolvimento consideram pertinente a compreensão interativa entre o processo de desenvolvimento e os ambientes em que se vive. O ser humano é aí entendido como aquele que influencia e é influenciado pelo cenário físico e social, em uma relação de bidirecionalidade. Como exemplos de pesquisa nesse campo, destacam-se os temas que tratam de ambientes de moradia no processo de envelhecimento; privacidade e independência ambiental; apego ao lugar e lugares favoritos como formas de possibilitar experiências de restauração (Günther et al., 2003; Khoury \& Günther, 2008; Rivlin, 2003).

Em especial sobre o lugar favorito, Macedo, Oliveira, Günther, Alves e Nóbrega (2008) consideraram que este pode estimular modificações fisiológicas relacionadas a bem-estar e relaxamento, mudança de humor em sentido positivo, estímulo da atenção e contemplação dos próprios sentimentos, o que auxiliaria na otimização da qualidade de vida do indivíduo. Torna-se pertinente, portanto, a investigação acerca dos lugares que interferem no bem-estar e na qualidade de vida durante o processo de envelhecimento, seja este saudável ou patológico, e a posterior formulação de alternativas de intervenções na área da saúde (Pereira et al., 2006). Diante disso, este trabalho busca apresentar e discutir a interação pessoa-ambiente no processo do envelhecimento, sobretudo no que diz respeito ao lugar favorito. Para tanto, alguns conceitos utilizados na Psicologia Ambiental - como espaço físico, lugar e apego ao lugar - são também apresentados.

\section{Psicologia Ambiental: Espaço físico, lugar e apego ao lugar}

A Psicologia Ambiental empenha-se em compreender as construções de significados e sentimentos elaboradas pelo sujeito, em sua relação com o meio físico e social, e as manifestações comportamentamentais daí derivadas. 0 fator ambiente e o processo de interação pessoa-ambiente são, portanto, tratados por essa área, entendendo-se que o ser humano é sujeito que se constrói em sua relação com o meio e que tal elaboração é acompanhada de comportamentos que afetam o próprio ambiente, num movimento de retroalimentação. 0 ambiente físico torna-se, pois, importante ao desenvolvimento humano, representando outro elemento que contribui na definição do indivíduo na sociedade, modificando o comportamento, influindo em pensamentos, emoções, cognição, interações sociais, bem-estar físico e self (Günther et al., 2003).

De acordo com Speller (2005), espaço significa o ambiente físico em si, diferenciando-se do termo lugar, que se refere ao espaço físico dotado do significado atribuído por seus utilizadores. Pode-se considerar identidade de lugar, por sua vez, o conjunto de atitudes, valores, crenças e significados elaborados pelo sujeito a partir de sua relação com o meio 
(Günther et al., 2003; Speller, 2005). Já o apego ao lugar, conceito derivado da teoria da vinculação afetiva de John Bowlby (1989) e Mary Ainsworth (1979/1997), reflete a relação emocional estabelecida com lugares, caracterizada pelo desejo de proximidade com ambientes que satisfazem as necessidades físicas e psicossociais do indivíduo e reforçam sua identidade pessoal (Günther et al., 2003).

Uma das formas de se estudar o apego ao lugar é por meio do lugar favorito. Para Newell (1997), os indivíduos tendem a preferir lugares relacionados ao ambiente natural, com corpos de água e vegetação. Entretanto, lugares favoritos podem ser também ambientes construídos ou virtuais. Isto porque o que está em questão é a busca de experiências e sensações prazerosas, relaxamento, independência, interações sociais e reflexão, que atuam como fatores restauradores, favorecendo o enfrentamento de situações ameaçadoras ou experiências negativas. Além disso, a escolha do lugar favorito depende de faixa etária, sexo, cultura e interesses (Macedo et al., 2008).

Tem-se, assim, a possibilidade do espaço físico converter-se em um espaço significativo e afetivo ao indivíduo, repercutindo no desenvolvimento, na cognição e no comportamento (Corraliza, 2002). Tais conceitos (identidade de lugar e apego ao lugar) podem se relacionar, por exemplo, a estudos de lugares favoritos e seus efeitos sobre o bem-estar psicológico dos indivíduos (Corraliza, 2002; Günther et al., 2003).

\section{Método}

Para a investigação da relação lugar favorito e envelhecimento, foi realizada uma pesquisa, em julho de 2009, na base de dados BVS-PSI e no Journal of Environmental Psychology, com dois objetivos centrais: (a) apresentar os documentos selecionados a partir das palavras-chave lugares favoritos e envelhecimento, lugares favoritos e idosos, favorite places e aging, (b) dentre estes, analisar os documentos que mencionam no corpo do texto a relação lugar/ lugares favoritos no envelhecimento, explorando o conteúdo dos mesmos.

A base de dados BVS-PSI reúne documentos da área de Psicologia, do Brasil e da América Latina, com acesso nos seguintes diretórios: Index
Psi Periódicos Técnico-Científicos, Index Psi Periódicos de Divulgação Científica, Index Psi Teses, Index Psi Livros, LILACS (Literatura Latinoamericana e do Caribe em Ciências da Saúde), Google Acadêmico, Index Psi TCC (Trabalhos de Conclusão de Cursos), Index Psi Monografias de Conclusão de Cursos de Especialização, PePSIC (Periódicos Eletrônicos em Psicologia), SciELO, Literatura Científica, Sites e Biografias em Psicologia. Para este trabalho foram excluídos os resultados do Google Acadêmico por causa da heterogeneidade dos achados. O Journal of Environmental Psychology é um periódico voltado a estudos e disciplinas com interesse na relação entre a pessoa e seu meio sociofísico, principalmente com foco na disciplina de Psicologia Ambiental.

\section{Resultados}

Na base de dados BVS-PSI não foram encontrados resultados nos seguintes diretórios: Index Psi Periódicos Técnico-Científicos, Index Psi Periódicos de Divulgação Científica, Index Psi Teses, Index Psi Livros, Index Psi TCC (Trabalhos de Conclusão de Cursos), Index Psi Monografias de Conclusão de Cursos de Especialização), e PePSIC (Periódicos Eletrônicos em Psicologia. Apenas o trabalho de Macedo et al. (2008) foi encontrado nessa base, sendo ele indexado tanto no LILACS quanto no SciELO.

0 tema central do documento encontrado na BVS-PSI, com o título $O$ lugar do afeto, o afeto pelo lugar: $O$ que dizem os idosos?, envolve a relação lugares favoritos/envelhecimento e, em específico, retrata os lugares favoritos, os lugares evitados e os afetos a eles relacionados em idosos. No momento do estudo, os autores eram vinculados no Brasil às Universidade de Brasília e Universidade Federal do Rio Grande do Norte e, no Reino Unido, ao OPENspace Research Centre, Edinburg College of Art.

$\mathrm{Na}$ pesquisa realizada no Journal of Environmental Psychology, foram encontrados 12 estudos. Os documentos foram separados em dois grupos: (a) estudos em que não é evidente a relação lugar/ lugares favoritos no envelhecimento, (b) estudos que mencionam a relação lugar/lugares favoritos no envelhecimento. A Tabela 1 relaciona os trabalhos incluídos no primeiro grupo. 
Tabela 1 - Estudos sem evidência da relação lugares favoritos no envelhecimento encontrados no Journal of Environmental Psychology

\begin{tabular}{lcl}
\hline Autor & Ano & Título \\
\hline Dixon \& Durrheim & 2004 & $\begin{array}{l}\text { Dislocating identity: Desegre- } \\
\text { gation and the transforma- } \\
\text { tion of place. }\end{array}$ \\
\hline Fraine et al. & 2007 & $\begin{array}{l}\text { At home on the road? Can } \\
\text { drivers' relationships with } \\
\text { their cars be associated with } \\
\text { territoriality? }\end{array}$ \\
\hline $\begin{array}{l}\text { Jorgensen \& } \\
\text { Stedman }\end{array}$ & 2001 & $\begin{array}{l}\text { Sense of place as an attitude: } \\
\text { Lakeshore owners attitudes } \\
\text { toward their properties. }\end{array}$ \\
\hline $\begin{array}{l}\text { Mazumdar \& } \\
\text { Mazumdar }\end{array}$ & 2009 & $\begin{array}{l}\text { Religion, immigration, and } \\
\text { home making in diaspora: }\end{array}$ \\
& & $\begin{array}{l}\text { Hindu space in Southern } \\
\text { California. }\end{array}$ \\
\hline Min \& Lee & 2006 & $\begin{array}{l}\text { Children's neighborhood } \\
\text { place as a psychological and } \\
\text { behavioral domain. }\end{array}$ \\
\hline Rechavi & 2009 & $\begin{array}{l}\text { A room for living: Private and } \\
\text { public aspects in the expe- } \\
\text { rience of the living room. }\end{array}$ \\
\hline
\end{tabular}

Fonte: Dados da pesquisa.

De forma geral, esses estudos não mencionam, no corpo do texto, a possível relação lugar/lugares favoritos no envelhecimento. Como exemplo, o estudo de Rechavi (2009), que apresentou achados sobre o uso e as experiências de pessoas na sala de estar. Mazumdar e Mazumdar (2009) retrataram a maneira como imigrantes decoram e criam suas casas em outro lugar, baseando-se na religião. Fraine et al. (2007) investigaram a relação entre as pessoas e seus carros no âmbito da teoria de Altman, sobre territorialidade humana. Com isso, examinaram descrições dadas por pessoas com diferentes formas de apropriação e utilizações de seus carros, explorando a congruência entre estas e as características utilizadas para descrever territórios humanos. No estudo de Min e Lee (2006), buscou-se a relação entre a fase de desenvolvimento infantil e o lugar, tratando-se de descrever as características positivas deste último para a criança. Já Dixon e Durrheim (2004), a partir de entrevistas realizadas em uma praia multirracial na África do Sul, discutiram conceitos como lugar e mudança de identidade de lugar. Defenderam a hipótese de que a diferença racial pode modificar a relação entre um indivíduo e o outro e entre indivíduo e lugar. 0 estudo de Jorgensen e Stedman
(2001) buscou discutir a teoria da atitude como base para o desenvolvimento do sentido de lugar. 0 objetivo desse estudo, bem como sua análise e discussão teórica não citaram a relação entre idosos/envelhecimento e lugar ou ambiente. Em contrapartida, a Tabela 2 relaciona estudos que mencionam no corpo do texto a relação lugar/lugares favoritos no envelhecimento.

Tabela 2 - Estudos com evidência da relação lugares favoritos no envelhecimento, encontrados no Journal of Environmental Psychology

\begin{tabular}{lcl}
\hline Autor & Ano & Título \\
\hline $\begin{array}{l}\text { Cloutier-Fisher } \\
\text { \& Harvey }\end{array}$ & 2009 & $\begin{array}{l}\text { Home beyond the house: Expe- } \\
\text { riences of place in an evolving } \\
\text { retirement community. }\end{array}$ \\
\hline Gross \& Lane & 2007 & $\begin{array}{l}\text { Landscapes of the lifespan: } \\
\text { Exploring accounts of own } \\
\text { gardens and gardening. }\end{array}$ \\
\hline Manzo & 2003 & $\begin{array}{l}\text { Beyond house and haven: To- } \\
\text { ward a revisioning of emotional } \\
\text { relationships with places. }\end{array}$ \\
\hline Oswald et al. & 2006 & $\begin{array}{l}\text { Homeward bound: Introducing } \\
\text { a four-domain model of percei- } \\
\text { ved housing in very old age. }\end{array}$ \\
\hline $\begin{array}{l}\text { Scopelliti \& } \\
\text { Giuliani }\end{array}$ & 2004 & $\begin{array}{l}\text { Choosing restorative environ- } \\
\text { ments across the lifespan: } \\
\text { A matter of place experience. }\end{array}$ \\
\hline $\begin{array}{l}\text { Smaldone, } \\
\text { Harris \& Sanyal }\end{array}$ & 2005 & $\begin{array}{l}\text { An exploration of place as a } \\
\text { process: The case of Jackson } \\
\text { Hole, WY. }\end{array}$ \\
\hline
\end{tabular}

Fonte: Dados da pesquisa.

O conteúdo desses estudos será explorado na discussão deste trabalho, a fim de contemplar seus objetivos centrais. Como forma de avaliar a contribuição teórico-metodológica dos trabalhos para a Psicologia Ambiental, também foram observadas questões relativas à constituição disciplinar das equipes de pesquisadores e suas propostas de trabalho integrado, bem como as perspectivas teóricas, os paradigmas de investigação, os delineamentos metodológicos e os contextos de pesquisa - se naturais ou artificiais - adotados nos estudos.

\section{Discussão}

Uma das formas de as pessoas se relacionarem com seus ambientes é observada pelo modo como 
esses incidem nas reações afetivas e emocionais dos indivíduos, sendo explicado principalmente pelo fenômeno de lugar e ambientes favoritos. A relevância deste tema pode ser encontrada na possibilidade de se pensar espaços que propiciem experiências positivas, estimulando o bem-estar (Macedo et al., 2008; Peron, 2002).

Como exemplo, o estudo encontrado na BVS-PSI, de Macedo et al. (2008), mostra que os lugares favoritos podem servir como estratégias de regulação das emoções. Nessa pesquisa, 340 participantes com idades entre 60 e 90 anos, residentes em Brasília (Brasil), responderam individualmente a uma entrevista semiestruturada que objetivou identificar os lugares favoritos de idosos quando eles se sentem alegres e quando não se sentem alegres, os lugares evitados e as razões para tais escolhas. As respostas em relação à questão "Qual o lugar que você prefere estar quando se sente alegre?" foram agrupadas em quatro categorias: (a) ambiente facilitador de interação social - bares, restaurantes, festas, feiras, shopping $(\mathrm{n}=134)$; (b) a própria casa ( $\mathrm{n}=114$ ); (c) ambiente natural - chácaras, cachoeiras, fazenda, parques, praia e campo $(\mathrm{n}=46)$; e $(\mathrm{d})$ igreja $(n=46)$. Assim, os autores observaram que, quando os idosos entrevistados se sentiam alegres, preferiam ambientes que estimulassem a interação social, seguindo-se a preferência pela casa. Esse resultado sugere que tais ambientes proporcionam bem-estar, autonomia, paz, segurança e privacidade.

Contudo as respostas sobre a preferência por lugares quando não se sente alegre, corresponderam em sua maioria à casa, pela facilidade de se manter isolado e em privacidade. Ao ambiente residencial seguiram-se os ambientes sociais, representando uma estratégia de enfrentamento da situação emocional adversa. Em contrapartida, os lugares mais evitados quando não se está alegre são os de alta densidade social, agitados, lugares tristes e com multidão, sugerindo que excedem à capacidade de manejo ambiental (Macedo et al., 2008).

Lugares que possibilitam interação social e a própria casa podem se apresentar aos idosos como lugares favoritos, com capacidade restauradora, pois favorecem mudanças fisiológicas gerando alterações de humor no sentido positivo, equilíbrio da capacidade de atenção, relaxamento e contemplação dos próprios sentimentos (Macedo et al., 2008). Esse dado também é mencionado em todos os outros estudos encontrados no Journal of Environmental Psychology
(Cloutier-Fisher \& Harvey, 2009; Gross \& Lane, 2007; Manzo, 2003; Oswald et al., 2006; Scopelliti \& Giuliani, 2004; Smaldone, Harris \& Sanyal, 2005).

Na pesquisa de Cloutier-Fisher e Harvey (2009), da Universidade de Victoria, Canadá e da Universidade de British Columbia, Canadá, foi desenvolvida a ideia de "lar para além da casa", representando as interações sociais (comunidade) como facilitadores de bem-estar e apego ao lugar. Os autores realizaram um estudo qualitativo, com 25 indivíduos com mais de 55 anos, considerados idosos saudáveis e com boas condições financeiras, que se mudaram para uma região que tem se transformado rapidamente em uma comunidade de aposentados. 0 estudo visou a dois objetivos: descrever o significado de lugar, casa e comunidade para idosos, com base na Psicologia Ambiental, Gerontologia Ambiental e Geografia Humana; e discutir conceitos como significado de casa e sentimento de pertença para novos moradores da região.

Observaram que, para os idosos mais velhos, existe um espaço geográfico composto e interligado por domínios pessoais, sociais e físicos, sendo a casa física e a comunidade compreendidas como fronteiras dinâmicas e permeáveis. 0 significado da casa para os idosos teria relação principalmente com os domínios pessoais e sociais. Ou seja, os domínios sociais (representados pela conexão de uma pessoa a outra) e pessoais (identidade que promove reconhecimento pelos membros da comunidade) foram categorias mais associadas.

Em outro estudo, Gross e Lane (2007), da Universidade de Loughborough, Reino Unido, exploraram um local específico da casa: o jardim - em diferentes fases de desenvolvimento da pessoa (participantes com 18 a 85 anos de idade). Consideraram a casa como elemento fundamental na vida dos indivíduos, em que são constituídas relações sociais e com o ambiente físico. Referiram-se ao jardim da mesma forma, sendo esse um recurso da casa, considerado um elemento de território pessoal, que auxilia na aproximação com a natureza.

Os dados coletados nas entrevistas foram separados em temas como: o escapismo (positiva distração ou fuga de ansiedade e restrições da vida cotidiana demonstrando o importante papel do ar livre na regulação de estados mentais), a propriedade e a identidade, e relacionamentos. A maioria dos participantes resgatou o uso do jardim na infância de forma positiva, identificando-o como significativo e 
destacando-o pela conexão com a natureza. 0 papel do jardim na adolescência e na idade adulta precoce, ou seja, em indivíduos com menos de 30 anos de idade, não se demonstrou significativo, transparecendo percepções de que o jardim é um lugar para adultos mais velhos. Para os idosos, graças à debilitação da saúde ao longo do processo de envelhecimento, o cuidado do jardim parece ficar cada vez mais restrito, o que é apresentado como motivo de frustração e impacto diante da percepção de diminuição da capacidade física, com representações de luto, perda de identidade como jardineiros e perda de controle sobre o ambiente. Entretanto, graças à importância da conexão com a natureza representada pela jardinagem, houve referências a adaptações do modo de vida do idoso para prosseguir com atividades no jardim. Evidenciou-se no estudo que, para a maioria das pessoas, o significado do jardim está ligado a memórias da infância, com dimensões físicas e sociais.

Os pesquisadores Oswald et al. (2006) realizaram um estudo abrangendo três países europeus, com uma amostra de 1.223 idosos, que viviam sozinhos em residências de cidades urbanas. 0 objetivo foi medir e compreender a satisfação com a habitação, a usabilidade da casa, o significado da casa e as crenças de controle a ela relacionadas. Nesse estudo, o foco central foi o lugar, o envelhecimento e a identidade de lugar. Referiram-se à expressão usabilidade da casa como predominantemente desenvolvida no âmbito da terapia ocupacional, em que o foco é a funcionalidade do ambiente doméstico diante das atividades preferidas.

A casa representou o espaço que cumpre o objetivo de funções (como uso e abrigo) e o lugar que envolve significados pessoais, experiências, relações sociais, valores, cognições e emoções (Oswald et al., 2006), como também mencionaram Gross e Lane (2007). Oswald et al. (2006) observaram que os participantes idosos que consideraram suas casas como úteis para atividades diárias apresentaram laços significativos com a residência e se mostraram mais satisfeitos com as condições de habitação. Os autores não evidenciaram variabilidade significativa entre as respostas encontradas nos três países da Europa, embora antecedentes pessoais, ambientais e condições de alojamento pudessem variar. A usabilidade da casa e o significado da casa estiveram fortemente associados em todas as amostras, sugerindo que características do ambiente doméstico e sua experiência subjetiva relacionam-se fundamentalmente com a saúde e o bem-estar dos idosos, sendo a casa um dos lugares favoritos desse grupo de pessoas. Assim, o ambiente da habitação que gera independência e bem-estar mostra-se importante ao processo de envelhecimento, servindo como mediador para a idade avançada saudável, no enfrentamento da perda funcional e na adaptação ambiental (Macedo et al., 2008; Oswald et al., 2006).

Smaldone, Harris e Sanyal (2005), da Universidade de West Virginia, Mogantown, Estados Unidos, e da Universidade de Idaho, Moscow, Estados Unidos, desenvolveram um estudo centrado teoricamente nos conceitos de lugar e identidade de lugar. Entrevistaram 43 participantes de idades entre $23 \mathrm{e}$ 77 anos, que frequentavam um parque, a fim de explorar a concepção de lugar (neste caso, do parque) como um processo, examinando três dimensões: curso da vida, busca de sentimento, e compromisso com um lugar. Os autores observaram que 25 dos 43 indivíduos relataram que o lugar (parque) mostrava-se importante graças aos momentos partilhados com significados.

Neste estudo, são reforçadas as ideias de autores já citados (Cloutier-Fisher \& Harvey, 2009; Gross \& Lane, 2007; Oswald et al., 2006; Smaldone, Harris \& Sanyal, 2005), ao se mencionar que as pessoas utilizam ativamente e constantemente configurações físicas/ambientais para regular as emoções, frequentando lugares favoritos que geram bem-estar. Isso pode ser observado nas entrevistas, em que a grande maioria (29 indivíduos) relatou preferir ambientes naturais e sociais (tal como o parque), que podem apresentar qualidades restauradoras - proporcionando relaxamento, contemplação, inspiração e renovação - seguidos pela preferência da própria casa.

Na revisão de literatura de Manzo (2003), da Universidade de Washington, Estados Unidos, são discutidas temáticas como a relação bidirecional pessoa-lugar e emoção, residência e lugar, efeitos negativos e experiência no lugar, fenômenos dinâmicos do lugar, lugar como processo. No corpo do texto, são mencionados em dois momentos a relação idoso/envelhecimento e lugar/lugar favorito, ao se tratar: (a) da relação frequentar praças e idosos, (b) do fator histórico-social como possível preditor para a escolha do lugar para idosos, influenciando na relação entre pessoa e lugar/lugar favorito, principalmente ao se observar a história da política racial. Mencionaram o importante papel das praças públicas promotoras de encontros entre os idosos, 
que vivenciam parte do dia nesses locais, caracterizados como lugares favoritos que geram bem-estar.

0 ambiente natural e construído como lugar favorito é tema do estudo de Scopelliti e Giuliani (2004), do Instituto de Ciência Cognitiva e Tecnológica, Roma, Itália e da Universidade "La Sapienza", Roma, Itália. Os autores analisaram 67 entrevistas semiestruturadas com pessoas de três distintas fases da vida: jovens, adultos e idosos. As questões se referiram a experiências restauradoras que eles mesmos apresentavam durante o tempo livre do dia, durante a semana, e durante as férias, como também no dia de trabalho, na semana de trabalho e durante um longo período de trabalho, solicitando respostas que explicitassem as situações consideradas restauradoras. Foi elaborado também um check-list sobre experiências restauradoras, para que as pessoas selecionassem, de acordo com seu próprio julgamento, o grau de relaxamento e excitação de determinada situação. Os resultados mostraram que ambientes naturais e ambientes construídos podem ter diferentes potencialidades restaurativas para cada fase de vida.

A teoria da capacidade restauradora dos lugares, de Kaplan, fundamentou o trabalho desses autores. Como também descreveram Gross e Lane (2007), lugares restauradores geram prazer com recompensas psicológicas de bem-estar, atenção involuntária, descanso de fadiga mental decorrente do prolongado ou intensivo uso de atenção dirigida. Há quatro aspectos que caracterizam um ambiente como lugar de restauração: (a) being away (mudança de cenário e de experiência da vida diária); (b) fascinação (recuperação de fadiga mental a partir da atenção involuntária, sem esforço mental); (c) extensão (o ambiente é percebido como um todo no qual o conjunto de elementos está coerentemente relacionado); (d) compatibilidade (congruência entre as características do ambiente e as inclinações da pessoa) (Gross \& Lane, 2007; Scopelliti \& Giuliani, 2004). Para Gross e Lane (2007) e Macedo et al. (2008), ambientes que propiciam reflexão e bem-estar são base para a escolha do lugar favorito, fato que pode estar atrelado à capacidade desse lugar em proporcionar restauração.

De acordo com Scopelliti e Giuliani (2004), em virtude das alterações biológicas, fisiológicas, emocionais, de interesses e atividades, nas distintas fases de desenvolvimento, são possíveis mudanças quanto ao que as pessoas consideram ser uma experiência restauradora. Em todas as fases da vida, as pessoas se envolvem na busca pessoal e social de redefinição de identidade. Na idade adulta, tendem à preservação da integridade, utilizando estratégias adaptáveis inspiradas em experiências do passado, com estabilidade nos padrões de lazer e atividades. Tal estabilidade aumenta progressivamente com o envelhecimento, mas não significa ausência de necessidade de novidade e estimulação. 0 contexto social tem importante papel para a preferência do lugar e para a qualidade restauradora deste.

Resultados na pesquisa de Scopelliti e Giuliani (2004) mostraram uma caracterização diferente de experiências restauradoras para as distintas fases da vida. Jovens e adultos indicaram a preferência dos ambientes naturais como restauradores, com menos relatos sobre a casa. Os idosos indicaram preferir ambientes internos como restauradores, com mais frequência do que os ambientes naturais. As experiências restauradoras foram mais caracterizadas em termos de interação social para adultos do que para jovens e idosos, principalmente durante os finais de semana e férias, em analogia a um único dia. Relações com o parceiro foram significativamente mais importantes para adultos do que para os jovens; relações sociais com os familiares foram significativamente mais importantes para idosos; relações sociais com os amigos foram significativamente mais importantes para os jovens que para os dois outros grupos. Os quatro componentes propostos por Kaplan, sobre capacidade restauradora dos lugares, revelaram que a compatibilidade é o mais importante aspecto para a restauração em todas as faixas etárias deste estudo. Observou-se a importante relação entre restauração e componente social, restauração e relaxamento, restauração e emoção, evidenciando que os lugares favoritos geralmente possibilitam experiências restauradoras para todas as faixas etárias (Scopelliti \& Giuliani, 2004). Essa hipótese é compartilhada em todos os demais manuscritos encontrados e aqui discutidos.

Com o intuito de avaliar as contribuições teóricas e metodológicas da pesquisa envolvendo lugares favoritos no envelhecimento, foram também explorados, além de seus conteúdos, aspectos como a composição disciplinar das equipes de pesquisadores, as perspectivas teóricas, os paradigmas de investigação, os delineamentos metodológicos e os contextos de pesquisa adotados. Quanto às disciplinas dedicadas ao tema, estiveram presentes nos artigos revisados os departamentos de Arquitetura da Paisagem, 
Ciências Cognitivas e Tecnologia, Ciências Humanas, Silvicultura, Geografia e Centro sobre o Envelhecimento, Gerontologia e Ciências do Cuidado, Psicologia, Psicologia e Mudança Social, Psicologia Social e do Desenvolvimento, Pesquisa Psicológica do Envelhecimento, Recursos Naturais, e Terapia Ocupacional. 0 interesse, em maior ou menor grau, de diferentes domínios do conhecimento na relação lugar/ lugares favoritos e envelhecimento revela a natureza multidisciplinar dos estudos pessoa-ambiente.

Dos sete artigos analisados, quatro foram resultado da parceria entre dois ou mais campos disciplinares; um relatou a experiência de trabalho dentro de uma mesma área disciplinar, mas em centros universitários distintos; e apenas dois estudos, sendo um deles de revisão de literatura, foram produzidos por um único departamento. A integração disciplinar, evidentemente, exige o esforço de união entre diferentes linguagens, métodos e teorias. Sabe-se que ela não tem sido rápida ou fácil, mas já é possível perceber um movimento favorável à contraposição e ao entrosamento entre diferentes domínios, o que, como afirmou Moser (2005), poderá garantir uma resolução bem sucedida das questões ambientais.

Ao se tratar das perspectivas teóricas, por sua vez, faz-se necessário distinguir as duas abordagens que traduzem o movimento da Psicologia Ambiental ao longo dos anos. Se, em uma primeira fase, deu-se ênfase à pesquisa voltada às reações das pessoas a determinados estímulos, especialmente estímulos do ambiente construído, em um segundo momento, quando as temáticas envolvendo o ambiente natural ganharam destaque nos anos 1980, passou-se a compreender o indivíduo como figura ativa que, como argumenta Ittelson et al. (2005), intervém sobre o meio deliberada e autoconscientemente para atender às suas necessidades. 0 comportamento, que antes era tratado essencialmente como efeito, passou a ser considerado também causa das mudanças ambientais em curso. Atribuiu-se ao indivíduo, então, não somente a condição de vítima, mas a de agende causador de transformações (Kruse, 2004).

A mudança representou a passagem de um modelo tipicamente interacionista para um modelo sistêmico, transacionalista. Como explicou Valera (1996), o interacionismo na Psicologia Ambiental considera os aspectos ambiente e pessoa em uma relação de causa e efeito, em que o comportamento, ainda que mediado por características do indivíduo, é função do contexto ambiental. 0 movimento da relação é o da unidirecionalidade. Já no transacionalismo, pessoa e ambiente interagem dinamicamente: admite-se que não só o meio exerce influência sobre os indivíduos, mas os indivíduos, reciprocamente, exercem influência sobre o meio. É o movimento da bidirecionalidade. Assim, a Psicologia Ambiental passou de uma antropologia tradicionalmente reativa (reação a estímulos) a uma antropologia de tendência ativa e, acima de tudo, transacional.

Particularmente no que se refere à investigação dos lugares favoritos no processo de envelhecimento, observou-se uma tendência de natureza transacionalista, seguindo o movimento dos estudos da Psicologia Ambiental. A ênfase à bidirecionalidade da relação pessoa-ambiente pode ser percebida ao se considerar, por exemplo, o significado do lugar (efeito do ambiente sobre a pessoa) como aspecto relacionado a controle, uso e apropriação do espaço (efeito da pessoa sobre o ambiente).

Ao abordar os temas escapismo, propriedade e identidade, bem como relações, Gross e Lane (2007) discutiram tanto o efeito do jardim e da jardinagem sobre as pessoas, como a atenção destinada pelas pessoas ao espaço do jardim, em consequência do sentido de responsabilidade para com o lugar e do desejo de cuidado e manutenção. 0 comprometimento ambiental também foi estudado por Smaldone, Harris e Sanyal (2005) e relacionado à percepção de ser parte, ou seja, de pertencer ao lugar e a ele estar apegado. Scopelliti e Giuliani (2004), assim como Oswald et al. (2006), na mesma direção, enfatizaram que as atividades possibilitadas pelo ambiente (uso e controle do espaço) interferem no modo como as pessoas o avaliam e o percebem, e vice-versa, sugerindo um claro e contínuo movimento de bidirecionalidade, que vai da percepção à ação e da ação à percepção. Mesmo o estudo de Macedo et al. (2008), interessado no efeito do ambiente favorito sobre o idoso, reconheceu a importância de se abordar as atividades desenvolvidas nesses lugares, o que indica uma preocupação com a totalidade dos aspectos da relação pessoa-ambiente, sejam eles de natureza ativa ou reativa.

No que diz respeito aos paradigmas de investigação, entende-se, de acordo com Valera (1996), que a Psicologia Ambiental recente tem evitado abordagens individualistas para assumir cada vez mais um perfil social. Isso significa passar de uma perspectiva que privilegia uma análise individual com vistas à generalização e à busca de princípios universais 
de comportamento, para uma abordagem social, que entende a pessoa como parte de uma comunidade, definida e modulada por um contexto histórico-cultural específico.

A investigação dos lugares favoritos no envelhecimento parece também seguir essa tendência, ao referenciar seus achados mais em aspectos sociais que individuais. São evidências dessa perspectiva os estudos transculturais, que compreendem a existência de um background sociocultural para as relações humano-ambientais (Oswald et al., 2006); as pesquisas em comunidades específicas, assumindo-se que resultados diversos poderiam ser encontrados em diferentes contextos (Cloutier-Fisher \& Harvey, 2009; Scopelliti \& Giuliani, 2004); e, por fim, as abordagens qualitativas, cuja ênfase está nas percepções de um pequeno grupo de sujeitos e na compreensão de sua relação com o meio, com independência de aspectos ligados à replicabilidade para cenários diversos (Cloutier-Fisher \& Harvey, 2009; Gross \& Lane, 2007; Smaldone, Harris \& Sanyal, 2005).

Tratando-se da dimensão metodológica, por sua vez, cabe ressaltar que as condições de existência de uma investigação devem ser satisfeitas unicamente pela relação que se estabelece entre um sujeito curioso e um objeto. A pesquisa científica tem, pois, como ponto inicial de desdobramento o objeto/ fenômeno a ser investigado, e sua validade se sustenta pela adoção de sistemáticas que respondam a uma situação-problema, segundo critérios que perpassam, entre outros, o rigor e a coerência. Fundamentalmente, as perspectivas teórico-metodológicas adotadas deverão ser capazes de responder, com legitimidade, as perguntas do pesquisador.

A história e a epistemologia da Psicologia revelam um debate constante acerca do delineamento metodológico mais adequado à construção do conhecimento. Para a Psicologia Ambiental, o holismo é, sem dúvida, um critério importante nessa construção e diz respeito a uma postura que está sempre em busca de identificar a totalidade de fatores constituintes de uma situação em questão. Metodologicamente, esse critério se traduz por uma abordagem preocupada em capturar dados complementares que retratem o quadro geral em que estão inseridos o problema de pesquisa e seus atores, evitando as lacunas e os vieses possíveis das investigações que analisam dimensões parciais de um fenômeno.

Conforme discutiram Günther, Elali e Pinheiro (2008), no último capítulo do livro intitulado
Métodos de pesquisa nos estudos pessoa-ambiente, como cada método, em razão das limitações próprias que o definem, permite apenas uma reprodução incompleta da realidade, a Psicologia Ambiental tem se dedicado à chamada triangulação metodológica: articulação entre técnicas de pesquisa pertencentes a delineamentos metodológicos diferentes que possibilita averiguar com maior precisão a multiplicidade de dimensões e aspectos envolvidos em uma situação-problema. Ainda segundo esses mesmos autores, o fenômeno da interação pessoa-ambiente é demasiado complexo - haja vista a multiplicidade de disciplinas que o estudam - para ser abordado por uma ou outra tipologia metodológica.

Quanto às práticas de pesquisa da relação pessoa-ambiente no processo de envelhecimento, o uso de multimétodos ainda parece limitado. 0 método de levantamento de dados é dominante nesses estudos e, à exceção do trabalho de Oswald et al. (2006) que associou as técnicas de questionário e entrevista - todas as demais investigações fizeram uso exclusivo da entrevista semiestruturada. Não houve referência, por exemplo, a técnicas auxiliares de observação direta do comportamento - que possibilitam compreender aspectos da interação pessoa-ambiente dificilmente comunicados por meio de entrevistas e questionários, e minimizam o viés característico da interação social - tampouco práticas interrogativas como as autobiografias ambientais, a produção de imagens e modelos, e a classificação e ordenação de fotografias.

As autobiografias são textos produzidos pelos indivíduos pesquisados, sobre as suas experiências em relação a ambientes específicos. Em geral, revelam laços afetivos e cognitivos fundamentais à compreensão das relações estabelecidas entre as pessoas e os ambientes (Elali \& Pinheiro, 2008). Na produção de imagens e modelos, o participante elabora material não verbal, como fotografias, desenhos, mapas mentais e maquetes, que o possibilitam expressar percepções ambientais importantes, com base em experiências vividas ou esperadas. No caso da classificação e do ordenamento de fotografias, é o pesquisador que apresenta as imagens ao entrevistado, que recebe a tarefa de avaliá-las, agrupá-las e/ou ordená-las segundo critérios pré-estabelecidos ou criados pelo próprio respondente. 0 produto dessas diferentes técnicas permite acessar percepções e representações adicionais por meio de linguagens diversas da falada e escrita, abrindo 
novos campos de investigação para estudiosos da relação pessoa-ambiente.

Por fim, foi possível também verificar, nos estudos revisados, a preferência pela pesquisa em ambientes reais, fossem naturais ou construídos. Nenhum estudo fez uso de contextos artificiais ou simulados em computador, por exemplo. A tendência da pesquisa em cenários reais também acompanha a Psicologia Ambiental. Quando do seu nascimento, ao fim dos anos 1950, havia no meio acadêmico muita discussão acerca da relevância dos resultados obtidos em laboratórios (Valera, 1996). Para a Psicologia Ambiental, a artificialização de contextos ambientais impossibilitaria uma compreensão adequada do conjunto de variáveis atuantes na relação pessoa-ambiente em toda a sua complexidade.

\section{Considerações finais}

Apesar do declínio neural normal e/ou patológico durante o envelhecimento, a plasticidade cerebral age como ferramenta de interação ao meio, como forma de adaptação do cérebro às novas exigências físicas, sociais, psicológicas e ambientais, buscando compensar as dificuldades e os novos desafios do indivíduo (Cardoso, Japiassú, Cardoso \& Levandoski, 2007). 0 ambiente físico no qual o idoso está inserido pode auxiliar na independência ou dependência, na restauração mental/psicológica/física e na inserção social do indivíduo (Pereira et al., 2006).

A pesquisa bibliográfica aqui realizada revelou conceitos compartilhados como identidade de lugar, lugar favorito e capacidade restauradora dos lugares. Observou-se que a casa e os ambientes que favorecem as interações sociais geralmente caracterizam o lugar favorito do idoso, estimulando a experiência de bem-estar e restauração decorrentes da satisfação e do prazer vivenciado.

Contribuições importantes desses estudos para a Psicologia Ambiental puderam também ser percebidas, especialmente no que diz respeito a uma postura que ultrapassa os limites da multidisciplinaridade para se constituir cada vez mais inter/ transdisciplinar; e às perspectivas teóricas e aos paradigmas de investigação adotados, que exploraram a natureza transacional da relação pessoa-ambiente, em que o indivíduo foi entendido, não à parte, mas inserido em um contexto sociocultural específico. Desafios são lançados, entretanto, no sentido de uma maior proximidade à abordagem multimetodológica, que tem recebido especial atenção da Psicologia Ambiental. Por fim, faz-se necessário considerar a importância de se analisarem mais estudos interessados na relação lugar/lugar preferido e envelhecimento, por ventura disponibilizados em outras bases de dados, aqui não investigadas.

Esse estudo tem apoio financeiro da Coordenação de Aperfeiçoamento de Pessoal de Nível Superior (Capes).

\section{Referências}

Ainsworth, M. D. S. (1989). Attachments beyond infancy. American Psychologist, 44, 709-716.

Aversi-Ferreira, T. A., Rodrigues, H. G., \& Paiva, L. R. (2008). Efeitos do envelhecimento sobre o encéfalo. Revista Brasileira de Ciências do Envelhecimento Humano, 5(2), 46-64.

Bowlby, J. (1997). Formação e rompimento dos laços afetivos (3. ed.). São Paulo: M. Fontes. (Originalmente publicado em 1979).

Cardoso, A. S., Japiassú, A. T., Cardoso, L. S., \& Levandoski, G. (2007). 0 processo de envelhecimento do sistema nervoso e possíveis influências da atividade física. Publicatio UEPG Ciências Biológicas e da Saúde, 13(3/4), 29-44.

Cloutier-Fisher, D., \& Harvey, J. (2009). Home beyond the house: Experiences of place in an evolving retirement community. Journal of Environmental Psychology, 29(2), 246-255.

Corraliza, J. A. (2002). Emoción y ambiente. In J. I. Aragonés \& M. Amérigo (Coord.). Psicología ambiental (pp. 59-76). Madrid: Pirámide.

Dixon, J., \& Durrheim, K. (2004). Dislocating identity: Desegregation and the transformation of place. Journal of Environmental Psychology, 24(4), 455-473.

Elali, G. A., \& Pinheiro, J. Q. (2008). Autobiografia ambiental: Buscando afetos e cognições da experiência com ambientes. In J. Q. Pinheiro \& H. Günther (Org.). Métodos de pesquisa nos estudos pessoa-ambiente (pp. 217-251). São Paulo: Casa do Psicólogo. 
Fraine, G., Smith, S. G., Zinkiewicz, L., Chapman, R., \& Sheehan, M. (2007). At home on the road? Can drivers' relationships with their cars be associated with territoriality? Journal of Environmental Psychology, 27(3), 204-214.

Gross, H., \& Lane, N. (2007). Landscapes of the lifespan: Exploring accounts of own gardens and gardening. Journal of Environmental Psychology, 27(3), 225-241.

Günther, H., Elali, G. A., \& Pinheiro, J. Q. (2008). A abordagem multimétodos em estudos pessoa-ambiente: Características, definições e implicações. In J. Q. Pinheiro \& H. Günther (Org.). Métodos de pesquisa nos estudos pessoa-ambiente (pp. 369-396). São Paulo: Casa do Psicólogo.

Günther, I. A., Nepomuceno, G. M., Spehar, M. C., \& Günther, H. (2003). Lugares favoritos de adolescentes no Distrito Federal. Estudos de Psicologia, 8(2), 299-308.

Ittelson, W. H., Proshansky, H. M., Rivlin, L. G., \& Winkel, G. H. (2005). Homem ambiental. Série: Textos de Psicologia Ambiental, 14.

Jorgensen, B. S., \& Stedman, R. C. (2001). Sense of place as an attitude: Lakeshore owners attitudes toward their properties. Journal of Environmental Psychology, 21(3), 233-248.

Khoury, H. T. T., \& Günther, I. A. (2008). Ambiente de moradia e controle primário em idosos. Paidéia, 18(39), 53-60.

Kruse, L. (2004). Globalização e desenvolvimento sustentável como questões da Psicologia Ambiental. In E. T. O. Tassara, E. P. Rabinovich \& M. C. Guedes (Ed.). Psicologia e ambiente (pp. 133-142). São Paulo: EDUC.

Macedo, D., Oliveira, C. V., Günther, I. A., Alves, S. M., \& Nóbrega, T. S. (2008). O lugar do afeto, o afeto pelo lugar: 0 que dizem os idosos? Psicologia: Teoria e Pesquisa, 24(4), 441-449.

Manzo, L. C. (2003). Beyond house and haven: Toward a revisioning of emotional relationships with places. Journal of Environmental Psychology, 23(1), 47-61.

Mazumdar, S., \& Mazumdar, S. (2009). Religion, immigration, and home making in diaspora: Hindu space in Southern California. Journal of Environmental Psychology, 29(2), 256-266.
Min, B., \& Lee, J. (2006). Children's neighborhood place as a psychological and behavioral domain. Journal of Environmental Psychology, 26(1), 51-71.

Moser, G. (2005). Psicologia Ambiental e estudos pessoas-ambiente: Que tipo de colaboração multidisciplinar? Psicologia USP, 16(1/2), 131-140.

Newell, P. B. (1997). A cross-cultural examination of favorite places. Environment \& Behavior, 29, 495-514.

Oswald, F., Schilling, O., Wahl, H. W., Fänge, A., Sixsmith, J., \& Iwarsson, S. (2006). Homeward bound: Introducing a four-domain model of perceived housing in very old age. Journal of Environmental Psychology, 26(3), 187-201.

Pereira, R. J., Cotta, R. M. M., Franceschini, S. C. C., Ribeiro, R. C. L., Sampaio, R. F., Priore, S., et al. (2006). Contribuição dos domínios físico, social, psicológico e ambiental para a qualidade de vida global de idosos. Revista de Psiquiatria do Rio Grande do Sul, 28(1), 27-38.

Peron, E. (2002). Preferencias ambientales y capacidad restauradora de los lugares. In R. G. Mira, J. M. Sabucedo \& J. Romay (Ed.). Psicología e medio ambiente: Aspectos psicosociales, educativos y metodológicos (pp. 263-276). A Coruña: Universidad de A Coruña- Universidad de Santiago de Compostela.

Rechavi, T. B. (2009). A room for living: Private and public aspects in the experience of the living room. Journal of Environmental Psychology, 29(1), 133-143.

Rivlin, L. G. (2003). Olhando o passado e o futuro: Revendo pressupostos sobre as interrelações pessoa-ambiente. Estudos de Psicologia, 8(2), 215-220.

Scopelliti, M., \& Giuliani, M. V. (2004). Choosing restorative environments across the lifespan: A matter of place experience. Journal of Environmental Psychology, 24(4), 423-437.

Smaldone, D., Harris, C., \& Sanyal, N. (2005). An exploration of place as a process: The case of Jackson Hole, WY. Journal of Environmental Psychology, 25(4), 397-414.

Speller, G. M. (2005). A importância da vinculação ao lugar. In L. Soczka (Org.). Contextos humanos e psicologia ambiental (pp. 133-167). Lisboa: Fundação Calouste Gulbenkian.

Valera, S. (1996). Psicologia ambiental: Bases teóricas y epistemológicas. In L. Iñiguez \& E. Pol (Ed.). Cognición, representación y apropiación del espacio (pp. 1-14). Barcelona: Universidad de Barcelona Publicacions. 